\title{
Cardiac Tamponade: Epidemioclinical, Therapeutic and Evolutionary Aspects at the University Hospital of Brazzaville
}

\author{
Solange Flore Mongo Ngamami1 ${ }^{1,2}$, Armel Landry Batchi-Bouyou ${ }^{*}$, Evariste Pabingui ${ }^{1}$, \\ Christian Michel Kouala Landa1,2, Bertrand Fikahem Ellenga Mbolla1,2, \\ Jean Joseph Ekwa Sima1, Méo Stéphane Ikama ${ }^{1,2}$, Suzy-Gisèle Kimbally Kaky ${ }^{1,2}$ \\ ${ }^{1}$ Department of Cardiology, University Hospital of Brazzaville, Brazzaville, Republic of the Congo \\ ${ }^{2}$ Department of Doctoral Studies, Faculty of Health Sciences, Marien Ngouabi University, Brazzaville, Republic of the Congo \\ Email: *armel_b@hotmail.fr
}

How to cite this paper: Ngamami, S.F.M., Batchi-Bouyou, A.L., Pabingui, E., Landa, C.M.K., Mbolla, B.F.E., Sima, J.J.E., Ikama, M.S. and Kaky, S.-G.K. (2021) Cardiac Tamponade: Epidemioclinical, Therapeutic and Evolutionary Aspects at the University Hospital of Brazzaville. World Journal of Cardiovascular Diseases, 11, 572-582.

https://doi.org/10.4236/wjcd.2021.1112054

Received: October 25, 2021

Accepted: December 18, 2021

Published: December 21, 2021

Copyright $\odot 2021$ by author(s) and Scientific Research Publishing Inc. This work is licensed under the Creative Commons Attribution International License (CC BY 4.0).

http://creativecommons.org/licenses/by/4.0/

\section{(c) (i) Open Access}

\begin{abstract}
Objectives: Contribute to improving the management of cardiac tamponade in Congo. Background: Cardiac tamponade is an acute or subacute compression of the heart chambers by pericardial effusion responsible for uncertain prognosis for patients. The objective of this study is to help improve the management of patients with cardiac tamponade. Patients and Methods: We conducted a retrospective and descriptive study at the University Hospital of Brazzaville, from January 2015 to December 31, 2019. Included were all patients hospitalized for cardiac tamponade. Epidemioclinical, therapeutic and evolutionary data were analyzed. Results: An overall of 14 patients were divided into 9 men $(64.2 \%)$ and 5 women $(35.7 \%)$, i.e. a sex ratio of 1.8. The mean age of patients was $34.2 \pm 15.1$ years old (18 years to 64). The most represented comorbidity was hypertension $(\mathrm{n}=2 ; 14.3 \%)$. The most frequent clinical sign was dyspnea $(n=14 ; 100 \%)$. The ECG showed diffuse and concordant repolarization disturbances $(n=14 ; 100 \%)$, sinus tachycardia $(\mathrm{n}=13 ; 92.8 \%)$, microvoltage $(\mathrm{n}=12 ; 85.7 \%)$. Cardiomegaly was constant $(\mathrm{n}=14 ; 100 \%)$. In terms of transthoracic ultrasound, we found: diastolic compression of the right ventricle (RV) $(n=12 ; 85.7 \%)$, dilation of the inferior vena cava (IVC) $(\mathrm{n}=12 ; 85.7 \%)$. Treatment consisted of crystalloid vascular filling in all patients, pericardial puncture $(\mathrm{n}=7 ; 50 \%)$, surgical drainage $(n=12 ; 85.7 \%)$, anti-tuberculosis drugs $(n=11 ; 78,6 \%)$, antimitotics could be administered in one case $(n=1 ; 7.2 \%)$. Two cases of death were recorded, i.e. $14.3 \%$. Conclusion: Cardiac tamponade, although it is a rare condition, remains a serious pathology and difficult to manage in our context.
\end{abstract}




\section{Keywords}

Cardiac Tamponade, Clinical Presentation, Treatment, Congo

\section{Introduction}

Cardiac tamponade is an acute or subacute compression of the heart chambers by pericardial effusion [1]. It can potentially be a complication of any pericarditis [2] and be life-threatening for the patient. Cardiac tamponade, being a medical and/or surgical emergency, can be difficult to suspect clinically. The contribution of transthoracic ultrasound is essential for its diagnosis [3] [4]; the treatment, which should not be delayed, depends on the mechanism and the etiologies. Its frequency remains low around $2 \%$ to $3 \%$ of admissions according to hospital data [1].

In Congo, the frequency of cardiac tamponade is unknown. Although the management of emergency tamponade remains the same, its etiopathogenic mechanisms are numerous, requiring careful etiological research, with a view to better management and to avoid recurrence. It is with this in mind that we have carried out this present work, the general objective of which is to help improve the management of patients with cardiac tamponade.

\section{Patients and Methods}

We conducted a retrospective, descriptive study covering the period from January 2015 to December 2019, i.e. a period of five (5) years carried out in the Department of Cardiology and Internal Medicine, and the Cardiovascular Surgery Unit at the University Hospital of Brazzaville. Included were in our study patients aged over 18, hospitalized for cardiac tamponade, having a complete medical file, including a medical observation, a chest X-ray, an ECG, a cardiac ultrasound, a biological assessment (blood count, serum creatinine + clearance, blood ionogram, analysis of pericardial puncture fluid, ESR, CRP). Patients aged 18 and over admitted for a condition other than cardiac tamponade and those with an incomplete medical record were not included.

\subsection{Study Variables}

The following variables were studied: socio-demographic (age, sex, marital status, standard of living, level of education, socio-professional category, place of origin), clinics (hospitalization service, comorbidities, history of tuberculosis, tumor, radiation, Acute renal failure, pericarditis, system disease, flu syndrome, concept of surgery, alcoholism), concept of chest pain, Polypnea, Orthopnea, Tachypnea Anguish, Agitation, Profuse sweating, Bradypnea, Collapse, Paradoxical pulse, state of shock, spontaneous turgor of the jugular veins, pericardial friction, arterial pressure, heart rate, respiratory rate), paraclinical (ECG, chest $\mathrm{x}$-ray, echocardiography, biological assessment), therapeutic and progressive. 


\subsection{Statistical Analysis}

Our data was entered using Microsoft Excel version 2010 software, Epi-info 3.5.1 for database processing. The qualitative variables were expressed in number (n) and percentage (\%), the quantitative variables in means and standard deviations with the extremes.

\section{Results}

\section{Cardiac tamponade}

1) Socio-demographic data

Out of a total of 78 patients hospitalized for liquid pericarditis, 14 presented cardiac tamponade, i.e. a frequency of $0.2 \%$. The 14 patients selected for the study were divided into 9 men (64.2\%) and 5 women (35.7\%), for a sex ratio of 1.8. The mean age of the patients was $34.21 \pm 15.1$ years with ranges of 18 to 64 years. The most represented age group is that of $18-19$ years.

The socio-professional category was represented by the students represented $35.7 \%(n=5)$, civil servants $28.5(n=4)$, the liberal professions $21.4 \%(n=3)$, the unemployed 14, $3(\mathrm{n}=2)$.

The standard of living was low in $50 \%(\mathrm{n}=7)$ of the cases, medium in $43 \%$ ( $\mathrm{n}$ $=6)$ of the cases and high in $7 \%(\mathrm{n}=1)$.

\section{2) Medical history and comorbidities}

The medical history and the co-morbidities encountered were tuberculosis (28.6\%), hypertension (14.3\%), diabetes mellitus (7.1\%), tumors (7.1\%), Gougerot-Sjögren syndrome (7.1\%), pericarditis (7.1\%).

\section{3) Clinical data}

\section{a) Functional signs}

The distribution of patients according to the functional signs presented during cardiac tamponade is given in Table 1 .

\section{b) General signs}

Table 2 gives the distribution of patients according to the general signs presented by the patients during cardiac tamponade. It shows that low blood pressure (100\%) and tachycardia (100\%) were predominant general signs.

\section{c) Physical signs}

The distribution of patients according to physical signs during cardiac tamponade

Table 1. Distribution of functional signs presented by patients during cardiac tamponade.

\begin{tabular}{ccc}
\hline & Cases & Percentage \\
\hline Tachypnea & 14 & 100 \\
Polypnea & 14 & 100 \\
Thoracic pain & 11 & 78.6 \\
Cough & 11 & 78.6 \\
Orthopnea & 5 & 35.7 \\
Anguish/Agitation & 4 & 28.6 \\
\hline
\end{tabular}


reveals that right-sided heart failure signs and paradoxical pulse were predominant (Table 3).

\section{4) Paraclinical data}

\section{a) Electrocardiogram}

The distribution of patients according to the electrocardiographic data shows that diffuse concordant repolarization (92.8\%) and sinus tachycardia (92.8\%) were predominant abnormalities (Table 4).

Figure 1 shows an ECG of a patient on cardiac tamponade. It shows sinus tachycardia, microvoltage, and diffuse and concordant flat $\mathrm{T}$-wave repolarization disturbances.

\section{b) Chest X-ray}

The distribution of patients according to radiological abnormalities is presented in Table 5. Short and dilated arterial pedicle (100\%) and cardiomegaly (100\%) were predominant findings.

Table 2. Distribution of patients according to general signs.

\begin{tabular}{ccc}
\hline & Cases & Percentage \\
\hline Low Blood Pressure & 14 & 100 \\
Tachycardia & 14 & 100 \\
Fever & 10 & 71.4 \\
Pinch blood pressure & 3 & 21.4 \\
\hline
\end{tabular}

Table 3. Distribution of patients according to physical signs.

\begin{tabular}{ccc}
\hline & Cases & Percentage \\
\hline Signs of right-sided heart failure & 14 & 100 \\
Paradoxical pulse & 14 & 100 \\
Muffled heart sounds & 11 & 78.6 \\
State of shock & 10 & 71.3 \\
Collapsus & 9 & 64.2 \\
pericardial friction rub & 2 & 14.3 \\
\hline
\end{tabular}

Table 4. Distribution of patients according to electrocardiographic abnormalities.

\begin{tabular}{ccc}
\hline & Cases & Percentage \\
\hline Diffuse concordant repolarization abnormality & 13 & 92.8 \\
Sinus tachycardia & 13 & 92.8 \\
Microvoltage & 12 & 85.7 \\
LVH & 2 & 14.2 \\
Atrial fibrillation & 1 & 7.1 \\
Electrical alternans & 1 & 7.1 \\
\hline
\end{tabular}




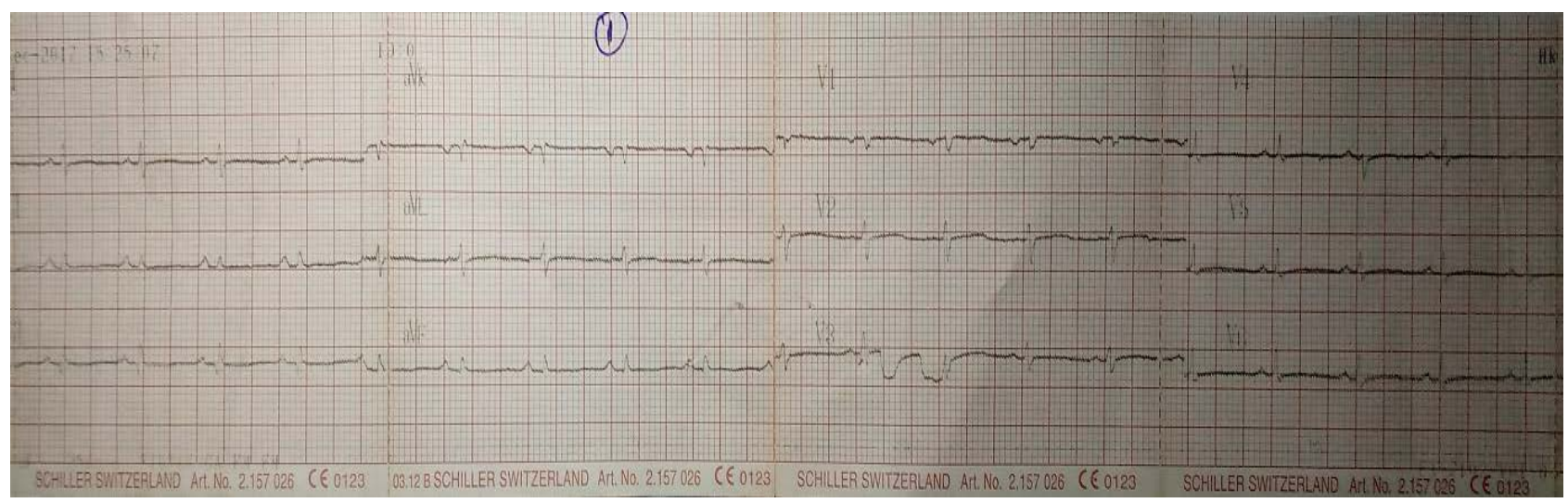

Figure 1. A standard 12-lead surface ECG of a cardiac tamponade patient with sinus tachycardia, microvoltage, and diffuse and concordant flat $\mathrm{T}$-wave repolarization disturbances.

Table 5. Distribution of patients according to radiological abnormalities.

\begin{tabular}{ccc}
\hline & Cases & Percentage \\
\hline Short and dilated arterial pedicle & 14 & 100 \\
Cardiomegaly & 14 & 100 \\
Heart shaped carafe & 9 & 64.3 \\
Elevation of the right diaphragmatic dome & 9 & 64.3 \\
Interstitial alveolar opacities & 6 & 42.8 \\
Associated pleural efusion & 4 & 28.6 \\
\hline
\end{tabular}

Figure 2 illustrates the image of a so-called carafe-shaped heart during cardiac tamponade. The frontal chest X-ray then shows cardiomegaly and a "carafe" appearance indicating the abundance of pericardial effusion (Figure 2).

\section{c) Cardiac doppler ultrasound}

The main echocardiographic data presented by patients receiving cardiac tamponade are listed in Table 6. Diastolic compression of the RV (85.7\%) and dilation of the IVC (85.7\%) are the most represented.

Figure 3 shows collapse of RV and Left atrium (LA) on transthoracic ultrasound by performing a two-dimensional apical four-chamber transthoracic ultrasound.

\section{5) Biological and anatomopathological data}

The distribution of patients according to biological data is shown in Table 7 representing $15.3 \%$ of patients with abnormal ESR.

The other biological and anatomopathological data were:

- Only one patient presented with severe anemia with a hemoglobin level of 3.7 $\mathrm{g} / \mathrm{dl}(7.2 \%)$;

- The puncture fluid is exudative in 11 patients $(78.6 \%)$;

- The tuberculin skin reaction is positive in 6 patients (42.8\%);

- In one patient a Koch Bacillus-type germ was isolated (7.2\%);

- The malignant cell was identified in one patient (7.2\%); 
- Antinuclear antibodies in autoimmune disease were positive in one patient (7.2\%);

- Retroviral serology performed in 10 patients was positive in two cases (14.3\%);

- The Gen Expert and the bacteriological examination of the sputum could not be performed.

\section{6) Etiologies of cardiac tamponade}

The different etiologies of cardiac tamponade: tuberculous (78.6\%), purulent (7.1\%), system disease (7.1\%), neoplastic (7.1\%).

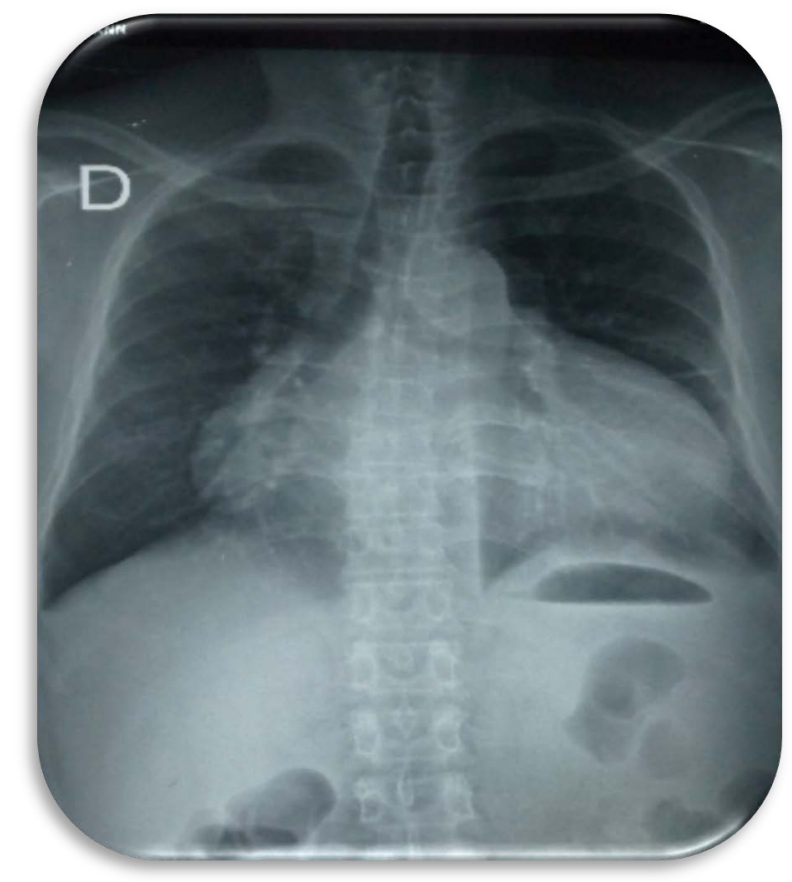

Figure 2. The frontal chest X-ray then shows cardiomegaly and a "carafe" appearance indicating the abundance of pericardial effusion.

Table 6. Distribution of patients according to echocardiographic abnormalities.

\begin{tabular}{ccc}
\hline & Cases & Percentage \\
\hline Diastolic compression of the RV & 12 & 85.7 \\
Dilation of the IVC & 12 & 85.7 \\
Proto and meso diastolic compression of the RV & 11 & 78.5 \\
Pericardial detachment $>$ to $20 \mathrm{~mm}$ & 10 & 71.4 \\
Presence of fibrin in the fluid & 8 & 57.1 \\
Swinging heart & 6 & 42.8 \\
Pericardial detachment 11 to $19 \mathrm{~mm}$ & 2 & 14.3 \\
Pericardial detachment $<$ to $10 \mathrm{~mm}$ & 2 & 14.3 \\
LV diastolic collapse & 1 & 7.1 \\
\hline
\end{tabular}




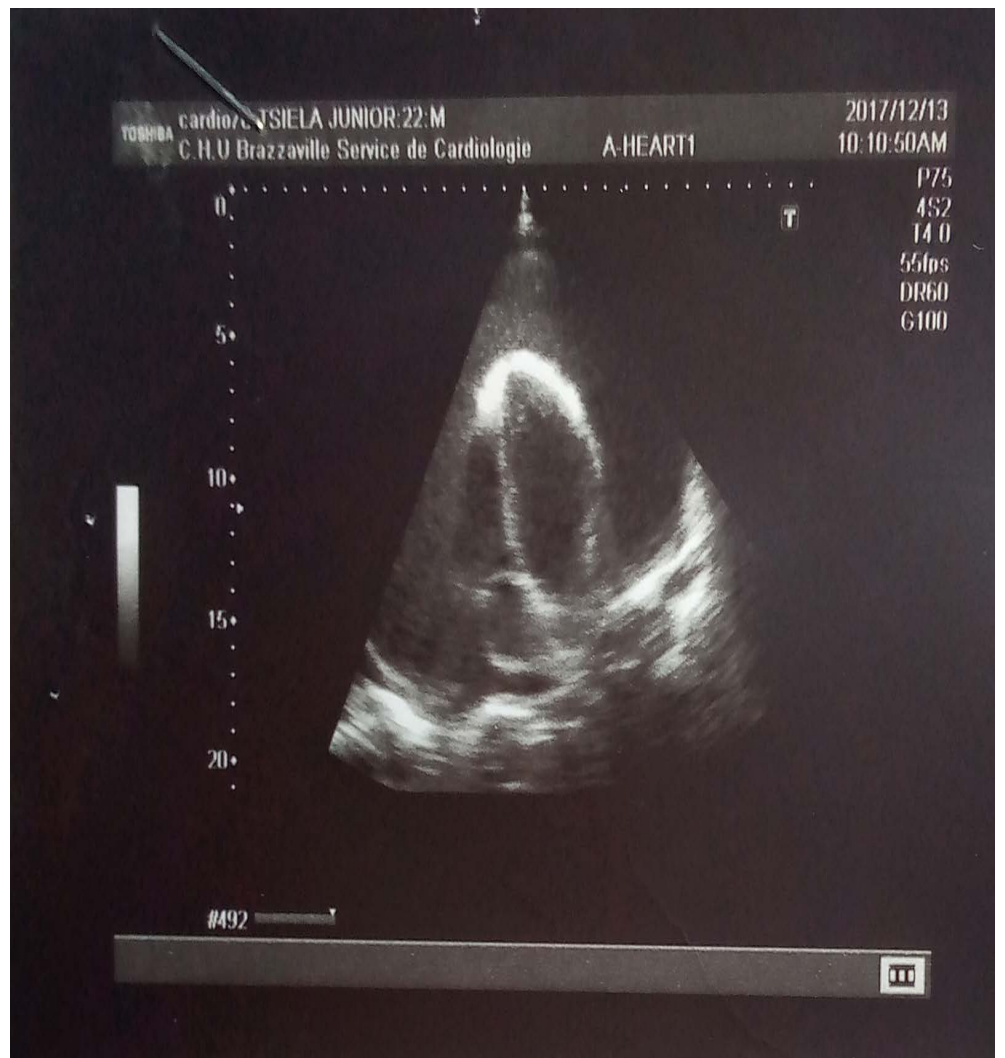

Figure 3. Two-dimensional apical four-chamber transthoracic ultrasound shows RV and LA collapse.

Table 7. Biological data of patients.

\begin{tabular}{ccc}
\hline & \multicolumn{2}{c}{ Cases (\%) } \\
\cline { 2 - 3 } & Normal & Abnormal \\
\hline ESR & $14(100)$ & $0(0.0)$ \\
C-Reactive Protein & $12(84.6)$ & $2(15.3)$ \\
Creatinemia & $14(100)$ & $0(0.0)$ \\
Glycemia & $14(100)$ & $0(0.0)$ \\
\hline
\end{tabular}

\section{7) Therapeutic data}

The various therapeutic means used for the management of cardiac tamponade are listed in Table 8. Vascular filling was performed in $100 \%$ of cases and surgical drainage in $85.7 \%$.

\section{8) Evolutionary data}

The mean hospital stay was $20.9 \pm 12.9$ days with extremes of 11 to 60 days. The outcome was considered favorable in 12 patients discharged without complications. Two cases of death were recorded, i.e. a case fatality of $14.3 \%$. The causes of these deaths were anemic shock (context of neoplastic tamponade) and cardio-circulatory arrest due to severe arrhythmia. 
Table 8. Therapeutic means used.

\begin{tabular}{ccc}
\hline & Cases & Percentage \\
\hline Vascular filling & 14 & 100 \\
Surgical drainage & 12 & 85.7 \\
Anti-tuberculosis treatment & 11 & 78.6 \\
Analgesic & 11 & 78.6 \\
Pericardial puncture & 7 & 50.0 \\
Vasopressor amines & 4 & 28.6 \\
Specific antibiotic & 4 & 28.6 \\
Corticosteroid therapy & 4 & 28.6 \\
Oxygen therapy & 2 & 14.3 \\
Antimitotic treatment & 1 & 7.2 \\
\hline
\end{tabular}

\section{Discussion}

\section{1) Socio-demographic aspects}

- Frequency:

The prevalence of pericardial diseases varies widely according to the population of interest: about $1.1 \%$ among people with cardiac complaints, between $3.3 \%$ and $6.8 \%$ among two large cohorts of patients with heart failure and up to $46.5 \%$ in an HIV-infected population with cardiac symptoms [5].

\section{- Gender:}

It appears in this study that cardiac tamponade was more common in men than women with 9 men to 5 women sex ratio of 1.8. This result is superimposable on that of Mehmet Kabukcu et al. who, for a series of 50 patients, had a male predominance with 34 men for 16 women [6].

-Age:

We also found in our study that the mean age of our patients was 34.21 years $( \pm 15.1)$ with the extremes of 18 and 64 years. Mehmet Kabukcu et al. found that the mean age was $51 \pm 17$ years (range, 12 - 80 years) [6]. This difference could be explained by the fact that these authors had taken into account pediatric cases in their study.

\section{2) Clinical aspects of tamponade}

In this study, we found 11 of the 14 patients, i.e. $78.6 \%$, who had presented with chest pain. While Salim Arous et al. in their study on cardiac tamponade: a rare manifestation of hypothyroidism found $50 \%$ of patients who presented with chest pain [7].

This difference in results is explained by the size of their sample, which was reduced to four (4) patients.

In our study, we found that tachypnea and polypnea are almost $100 \%$ present in all of our patients. Our results are similar to those found by Heierli B et al. with $92 \%$ of cases out of the 50 patients studied [8]. 
This finding is contrary to that of Bernhard Floerchinger et al., who found in 20 cases of delayed cardiac tamponade after open heart surgery noted dyspnea in 7 patients, i.e. $12 \%$ [9].

In our study, patients with a right sign represented $100 \%$ of cases while Bernhard Floerchinger et al. in their study of 20 cases on the delay of cardiac tamponade after open heart surgery noted: right heart failure (1 patient) or $5 \%$ [9]. This difference can be explained by the fact that these patients all underwent a cardiac intervention reducing the possibility of the appearance of the signs of right HF.

We found that all our patients had presented a paradoxical pulse, i.e. $100 \%$, while Chuttani $\mathrm{K}$ et al. found in a study "Diagnosis of cardiac tamponade after cardiac operation: Relative value of clinical, echocardiographic and hemodynamic signs" that the paradoxical pulse was noted in $48 \%$ of them [10]. This difference could be explained by the fact that all of these patients have already had heart surgery.

\section{3) Paraclinical aspects}

In our study, $92.96 \%$ of our patients presented with sinus tachycardia, however our patients who presented with microvoltage occupied $85.7 \%$ of the cases. This result can be superimposed on that of Salim Arous et al., who found similar values with sinus tachycardia of the order of $100 \%$ and microvoltage in $100 \%$ of cases [7].

In our study, from a radiological point of view, we found that all of our patients, i.e. $100 \%$, had presented with cardiomegaly. Our results are similar to that of Christopher L Roy et al. who in their study of articles that compared aspects of the clinical examination to a reference standard for the diagnosis of cardiac tamponade in 8 patients found $100 \%$ of cases of cardiomegaly on chest X-ray [11].

In our study, we noted that significant pericardial detachment and dilated IVC each accounted for $85.71 \%$ of cases in our study. These results are similar to that of Salim Arous et al. with $100 \%$ of cases of significant pericardial detachment and dilation of the IVC in $75 \%$ of cases [7].

The pericardial puncture fluid in our study was exudative in $78 \%$ of cases. This is simply because in our series tuberculosis is the dominant etiology. In addition, Alejandro Fernandez et al., in their study on cardiac tamponade in 40 patients with scleroderma, noted that the puncture fluid was exudative in $88 \%$ of cases [12]. This difference is explained by the fact that scleroderma is an inflammatory disease that can influence this result, while in our study several etiologies were represented.

\section{4) Etiologies}

Tuberculosis according to our study represented $78.6 \%$ of cases, systemic lupus erythematosus $7.2 \%$ and cancer $7.2 \%$ of cases. These results are different from those found by Allen KB et al. Found in a study of 50 patients treated from January 1998 to March 2002 for pericardial effusion and tamponade: cancer in 15 patients, i.e. (30\%), systemic lupus erythematosus in 2 patients or (4\%), tuberculosis in $1(2 \%)$. This difference can be explained by the fact that in Africa 
tuberculosis is an endemic where the frequency of contamination remains sharply increasing due to the outbreak of the HIV/AIDS epidemic [6].

\section{5) Therapeutic means used}

In our study, in our patients, surgical drainage accounted for $85.71 \%$ of cases. A result almost similar to that found by Allen $\mathrm{KB}$ et al., who in a retrospective study at Akdeniz University Hospital, performed a percutaneous catheter drainage treatment in 50 patients or $100 \%$ of cases [13].

We found that in our series the pericardial puncture was performed in $50 \%$ of our patients while Allen $\mathrm{KB}$ et al. performed in all patients, the pericardial puncture via the subxiphoid region, i.e. $100 \%$. This situation could be explained simply by the choice of a hospital technique [13].

\section{6) Prognosis of tamponade}

Tamponade is a serious complication of fluid pericarditis which can be life-threatening in patients. The observation bears witness to the results of our study: we found two (2) cases of death, i.e. 14.29\%. This observation is found in the literature. Indeed by Alejandro et al. who in their study on cardiac tamponade in 40 patients with scleroderma noted that five (5) patients, i.e. $12.5 \%$, had died during their hospitalization [12].

On the other hand, Allen KB et al. had 2 cases, i.e. (4\%) of patients who died in hospital in their study. This difference could be explained by the fact that the size of our study is smaller than that of Allen KB et al. [13].

\section{7) Limitation of the study}

There are some limitations of the study due to the small number of patients included in the study. This is due to a poor incidence of cardiac tamponade worldwide [5].

\section{Conclusion}

The frequency of cardiac tamponade remains low in hospitalization at the Brazzaville University Hospital. This pathology often affects young and male subjects. The diagnosis is clinical, the confirmation of which is based on the performance of the transthoracic ultrasound. The etiologies are many dominated by tuberculosis in our context. Its management consisted of evacuation of the liquid (puncture/drainage), associated with etiological treatment. In view of the foregoing, an emphasis will be placed on the diagnosis which must not suffer from any delay and urgent treatment in order to improve the prognosis, although the evolutionary modalities remain closely linked to the delay in taking charge and etiologies.

\section{Conflicts of Interest}

The authors declare no conflicts of interest regarding the publication of this paper.

\section{References}

[1] Reinmuller, R., Groll, R. and Lipton, M.J. (2004) CT and MR Imaging of Pericardial 
Desease. Radiologic Clinics of North America, 42, 587-601.

https://doi.org/10.1016/j.rcl.2004.03.003

[2] Georges, J., Tabone, X., Metzger, J.P. and Vacheron, A. (1992) Tamponnade sans rupture cardiaque après thrombolyse itérative dans l'infarctus aigü de myocarde. Archives des Maladies du Coeur et des Vaisseaux, 85, 1343-1346.

[3] Troughton, R.W., Asher, C.R. and Klein, A.L. (2004) Pericarditis. Lancet, 363, 717-727. https://doi.org/10.1016/S0140-6736(04)15648-1

[4] Dormager, V. (1994) Comment diagnostiquer une tamponnade? Réalités Cardiologiques, 68, 22-25.

[5] Noubiap, J.J., Agbor, V.N., Ndoadoumgue, A.L., Nkeck, J.R., Kamguia, A., Nyaga, U.F., et al. (2019) Epidemiology of Pericardial Diseases in Africa: A Systematic Scoping Review. Heart, 105, 180-188. https://doi.org/10.1136/heartjnl-2018-313922

[6] Kabukcu, M., Demircioglu, F., Yanik, E., Basarici, I. and Ersel, F. (2004) Pericardial Tamponade and Large Pericardial Effusions: Causal Factors and Efficacy of Percutaneous Catheter Drainage in 50 Patients. Texas Heart Institute Journal, 31, 398-403.

[7] Arous, S., Ettaoumi, Y., Najih, H., Makani, S., Alami, A.A. and Habbal, R. (2015) La tamponnade cardiaque: Une manifestation rare de l'hypothyrö̈die. The Pan African Medical Journal, 22, Article No. 104. https://doi.org/10.11604/pamj.2015.22.104.7941

[8] Heierli, B., Anderes, U. and Follath, F. (1981) Diagnosis and Therapy of Cardiac Tamponade: An Analysis of 50 Patients. Schweizerische medizinische Wochenschrift, 111, 735-741. (In German)

[9] Floerchinger, B., Camboni, D., Schopka, S., Kolat, P., Hilker, M. and Schmid, C. (2013) Delayed Cardiac Tamponade after Open Heart Surgery Is Supplemental CT Imaging Reasonable? Journal of Cardiothoracic Surgery, 8, Article No. 158. https://doi.org/10.1186/1749-8090-8-158

[10] Chuttani, K., Tischler, M.D. and Pandian, N.G. (1994) Diagnosis of Cardiac Tamponade after Cardiac Surgery: Relative Value of Clinical, Echocardiographic, and Hemodynamic Signs. American Heart Journal, 127, 913-918. https://doi.org/10.1016/0002-8703(94)90561-4

[11] Roy, C.L., Minor, M.A., Brookhart, M.A. and Choudhry, N.K. (2007) Does This Patient with a Pericardial Effusion Have Cardiac Tamponade? JAMA, 297, 1810-1818. https://doi.org/10.1001/jama.297.16.1810

[12] Fernández Morales, A., Iniesta, N., Fernández-Codina, A., Vaz de Cunha, J., Pérez Romero, T., Hurtado García, R., Simeón-Aznar, C.P., Fonollosa, V., Cervera, R. and Espinosa, G. (2017) Cardiac Tamponade and Severe Pericardial Effusion in Systemic Sclerosis: Report of Nine Patients and Review of the Literature. International Journal of Rheumatic Diseases, 20, 1582-1592. https://doi.org/10.1111/1756-185X.12952

[13] Allen, K.B., Faber, L.P., Warren, W.H. and Shaar, C.J. (1999) Pericardial Effusion: Subxiphoid Pericardiostomy versus Percutaneous Catheter Drainage. The Annals of Thoracic Surgery, 67, 437-440. https://doi.org/10.1016/S0003-4975(98)01192-8 